\title{
La influencia del Sol en la Tierra y otros planetas: Clima espacial
}

\author{
The Sun's influence on Earth and other planets: Space weather \\ Jenny Marcela Rodríguez Gómez ${ }^{* 1]}$ \\ ${ }^{1}$ Skolkovo Institute of Science and Technology, Space Center, Moscow, Russian Federation.
}

\begin{abstract}
Recibida en 28 de Noviembre, 2020. Revisado en 15 de Marzo, 2021. Aceptado en 19 de abril, 2021.
La actividad magnética del Sol domina fenómenos a través del espacio, esto incluye la interacción con los planetas, específicamente sus magnetosferas e ionosferas. Estas interacciones son descritas a través de una disciplina llamada clima espacial. Este artículo aborda una descripción del viento solar, las magnetosferas planetarias y eventos de clima espacial extremo en la Tierra.
\end{abstract}

Palabras clave: Sol, planetas, magnetosferas, clima espacial.

The Solar magnetic activity dominates phenomena through space. The Solar interaction with planets, their magnetospheres and ionospheres are described through a discipline called space weather. This article addresses a description of the solar wind, planetary magnetospheres, and extreme space weather events at Earth.

keywords: Sun, planets, magnetosphere, space weather.

\section{Introducción}

El Sol influye en el entorno espacial de la Tierra. Los impactos tecnológicos y sociales de esa interacción son muy importantes en nuestra sociedad. Su influencia se extiende a otros planetas. Estas interacciones generan diferentes fenómenos dependiendo de las características de cada planeta.

El sol es una estrella que posee una estructura estratificada compuesta por un núcleo, una zona radiativa, una zona convectiva, la fotosfera, la cromosfera, la región de transición y la corona solar [1, 2]. Las manchas solares fueron las primeras características que se observaron en el Sol a simple vista. Las manchas solares pueden ayudar a caracterizar la actividad magnética solar. En general, la actividad solar puede ser caracterizada por la aparición de manchas solares en ciclos de $~ 11$ años, en los que se registran periodos de gran actividad en donde más manchas solares aparecen en la superficie del Sol en comparación con otros periodos de inactividad donde algunas o ninguna mancha aparece en la superficie solar [1, 3, 4].

La actividad magnética del Sol domina varios eventos eruptivos como llamaradas (flares) y las eyecciones de masa coronal (Coronal Mass Ejections (CMEs)) [5], así como las variaciones en el viento solar provenientes de la corona solar o región más externa del Sol (Figura 1). El viento solar proveniente de los huecos coronales (coronal holes), es definido como el flujo de partículas cargadas (electrones e iones) liberadas desde la corona solar [7, 8].

\footnotetext{
${ }^{*}$ Correo electrónico: jemfisi@gmail.com
}

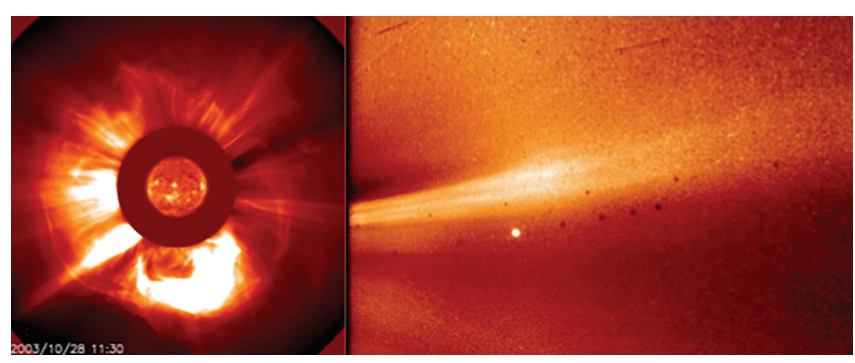

Figura 1: Panel izquierdo: Eyección de masa coronal (Coronal Mass Ejections (CMEs)) 28 de Octubre de $2003^{11}$ fue observada por el instrumento LASCO a bordo del satélite SOHO [6]. Panel derecho: viento solar en la corona solar visto con el instrumento WISPR de la sonda Solar Parker ${ }^{2}$

Estas partículas interactúan con los planetas formando fenómenos como la aurora debido a la interacción con el campo magnético de cada planeta. La interacción entre el viento solar y el campo magnético de la Tierra, generan transferencia de energía y momento desde el Sol dentro de nuestro entorno espacial [9 14]. El viento solar proporciona claves que permiten enlazar la atmósfera solar y el entorno de los planetas.

La Figura 1 muestra un ejemplo de un gran evento solar, este ocurrió el 28 de Octubre de 2003 fue originado desde la región activa 10486, un gran llamarada de clasificación X 17.2 desencadenó un fuerte evento de protones de alta energía, junto con una eyección de

1 https://sohowww.nascom.nasa.gov/data/archive.html

2 https://physicsworld.com/a/nasa-mission-touches-the-edge-ofthe-sun/ 
masa coronal (CME) rápida que golpeó la Tierra el 29 de Octubre, este evento es ampliamente conocido como parte de las tormentas solares de Halloween en 2003 (panel izquierdo). Región de viento solar en una región de la corona solar visto con el instrumento WISPR de la sonda Solar Parker lanzada en 2018, nombrada como "la misión para tocar el Sol", el objetivo de la misión es estudiar la atmósfera solar y el viento solar (panel derecho).

La interacción Sol-Tierra es fundamental y ante el creciente uso de tecnología, entender este proceso se torna muy importante. Una disciplina relativamente nueva se dedica al estudio de las condiciones del Sol a través del espacio y su interacción con los planetas, específicamente sus magnetosferas e ionosferas, es llamada clima espacial [15, 16]. El clima espacial describe variaciones a corto plazo en las diferentes formas de actividad solar y sus efectos en el entorno cercano a la Tierra [17]. Algunas definiciones de clima espacial han sido propuestas, por the US National Space Weather Plan (1995, 2000), the US National Space Weather Program ${ }^{3}$, una definición aceptada por 24 países en 2008 [18] y la más reciente propuesta por the World Meteorological Organisation (WMO ${ }^{4}$ En general todas coinciden en que el clima espacial alrededor de un planeta depende del viento solar, la intensidad del campo magnético interplanetario y del campo magnético del planeta.

Las principales características que determinan las condiciones del clima espacial alrededor de un cuerpo en el sistema solar son: la distancia entre el cuerpo y el Sol, las propiedades del campo magnético interplanetario (sus siglas en inglés $I M F)$. Así como la presencia de una densa atmósfera, en el caso de lunas planetarias gigantes, la existencia de una fuerte o débil magnetosfera. También puede ser afectado por la presencia de un cuerpo que genere su propio campo magnético, otras fuentes como plumas o volcanes y rayos cósmicos galácticos [19.

Este artículo aborda una perspectiva histórica del viento solar (sección 2), su origen (sección 3), descripción de las magnetosferas planetarias de planetas magnetizados y no magnetizados (sección 4), una descripción de clima espacial extremo en la Tierra (sección 5) y finalmente una discusion.

\section{Viento Solar Una Perspectiva Histórica}

Ludwig Biermann tratando de entender por qué la cola de un cometa se dirige hacia afuera desde el sol, propuso en 1951 que una radiación solar corpuscular relacionada con el campo magnético solar de gran escala podría estar detrás del fenómeno observado en la cola

\footnotetext{
$3 \longdiv { \text { http://www.nswp.gov/ } }$

4 Four year plan for WMO coordination of space weather activities, Toward a Space Weather Watch, Annex to draft esolution 4.2.2(2)/1 (cg-17, 2015), disponible en https://library.wmo.int/do c_num.php?explnum_id=3138
}

del cometa [20]. Esta idea plantearia la posibilidad de que el Sol pudiera estar liberando algún tipo de material fuera de él. Años después la existencia de viento solar fue descrita teóricamente por Eugene Parker entre 1950 y 1960. Parker propuso que la atmósfera del Sol no es estática sino altamente dinámica, además con las temperaturas altísimas en la corona solar que alcanzarían millones de Kelvins, todo estaría hirviendo y saldría algo de flujo 21. Ahora sabemos que el Sol emite un flujo de plasma llamado viento solar, que contiene principalmente electrones, protones y partículas alfa. Este flujo se emite desde la corona (la atmósfera superior solar) e interactúa con todas las partes del sistema solar, incluida la Tierra [15].

Desde la formación del sistema solar se puede rastrear la aparición de los primeros vestigios de viento solar masivo. Una característica importante del Sol joven durante los primeros $3 \times 10^{8}$ años es la presencia de un fuerte campo magnético comparado con el que observamos hoy. La combinación entre rápida rotación del Sol joven y su fuerte campo magnético permite el transporte de momento a través de la heliosfera [22 [24]. Actualmente el viento solar más tenue transporta momento angular o masa desde el Sol y este continúa dominando las condiciones del medio interplanetario, generando la espiral de campo magnético que impacta la magnetosfera terrestre de otros planetas de nuestro sistema solar [7] (Figura 2). El término magnetosfera fue descrito a mediados de 1900 s por Gold. La magnetosfera fue descrita como la región arriba de la ionosfera en la cual el campo magnético de la Tierra domina el movimiento del gas y las partículas cargadas. Esta región se extiende hasta una distancia de aproximadamente 10 radios de la Tierra [26].

La interacción del viento solar con la magnetosfera terrestre, se ve reflejada en las variaciones de la

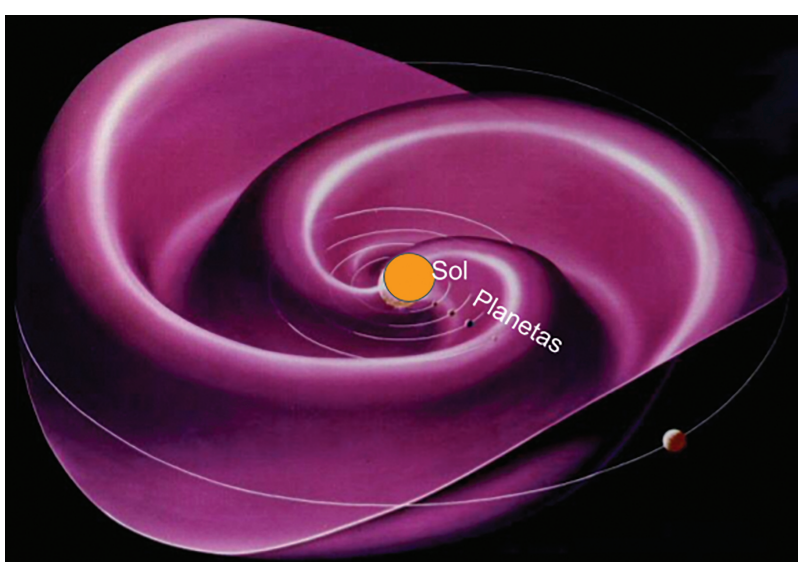

Figura 2: Estructura espacial del viento solar, es la estructura más grande del Sistema Solar, resultado de la influencia del campo magnético del Sol en el plasma del medio interplanetario, conocido como viento solar. Esta forma de espiral ondulada es conocida como la espiral de Parker y se ha comparado con la falda de una bailarina. Adaptada desde [25]. 


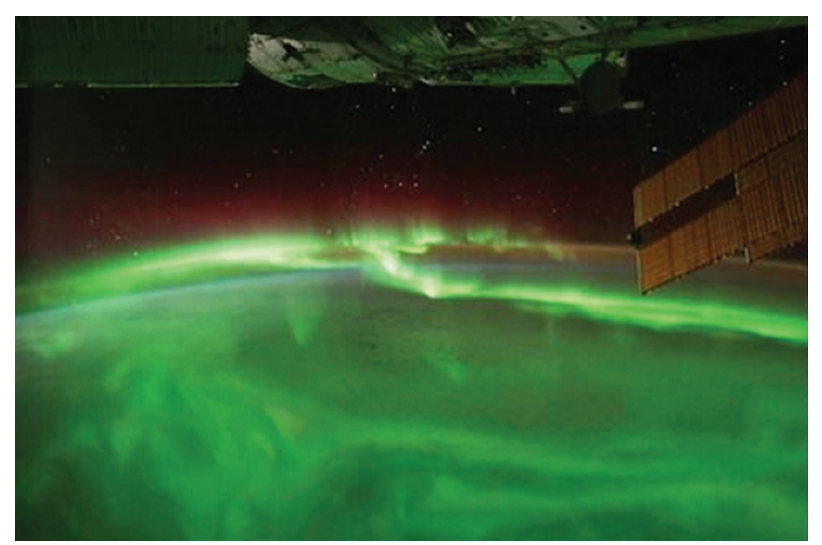

Figura 3: Imagen de la aurora tomada desde la Estación Espacial Internacional cuando cruzaba el sur del Océano Índico el 17 de septiembre de 2011. Fuente: [27].

actividad geomagnética. La actividad geomagnética ha sido estudiada, a través de las fluctuaciones del campo magnético, por ejemplo, Gilbert y Graham, en 1724 observaron cómo el movimiento de una aguja indicaba fluctuaciones en el campo magnético de la Tierra. En 1754 Frenchman Jean-Jacques d'Ortour de Mairan hizo las primeras mediciones aproximadas de la altura de las auroras. En general, en el siglo 18 un gran número de observaciones comenzaron a iluminar los orígenes de la aurora (Figura 3) 11.

En Septiembre de 1859, el astrónomo Richard Carrington observó un comportamiento anómalo en el Sol, que ahora conocemos como una fulguración solar (flare). Aproximadamente 17 horas después, sus efectos fueron observados en la Tierra, auroras ocurrieron en regiones de baja latitud, también los telégrafos presentaron fallas en Europa y Norteamérica 28.

Una idea del origen de la aurora polar debido a la interacción solar con el campo magnético de la Tierra fue sugerida por Birkeland. Él llevó a cabo tres expediciones en regiones polares entre 1896 y 1903, para confirmar su idea de que los disturbios magnéticos en la Tierra y la aurora boreal son debidas a rayos corpusculares emitidos por el Sol [29].

El astrónomo Edward Sabine tuvo un papel importante en las cruzadas magnéticas financiadas por la corona británica con el objetivo de estudiar el campo magnético terrestre. Al inicio del siglo XIX era ampliamente conocido que el campo magnético de la Tierra era altamente variable y afectaba los instrumentos de navegación [28].

En general, el modelo de dinamo es el más aceptado para explicar el origen del campo magnético de la Tierra, el tipo de dinamo $\alpha^{2}$ describe el campo magnético terrestre, este tipo de dinamo opera en el núcleo terrestre y considera que la rotación diferencial es casi despreciable. La intensidad de un campo magnético dipolar es dos veces más grande en el polo magnético que en el ecuador y decae rápidamente con la distancia, la intensidad del campo magnético en el ecuador disminuye con el cubo de la distancia [11]

$$
|B| \alpha\left(\frac{1}{r^{3}}\right)
$$

Satélites con instrumentos han medido la intensidad y dirección del campo magnético explorando alrededor de la Tierra y de otros planetas. Esas misiones han verificado el dipolo natural de la Tierra y de otros planetas con campo magnético como Mercurio, Júpiter, Saturno, Urano y Neptuno [11.

Podemos ver como desde el final del siglo XIX gracias a los grandes avances, descripciones físicas y experimentales tenemos algunas herramientas que permiten interpretar las fluctuaciones geomagnéticas. Ya en la era espacial se han dado avances sin precedentes a través de medidas in-situ que permitieron observar detalles del viento solar y de su interacción con el medio interplanetario. En 1962 la espacionave Mariner 2 dedicada principalmente a explorar Venus, detectó viento solar por primera vez. Esta exploración encontró viento solar con propiedades similares a las predichas por Parker (1958) 30 33.

En 1955, Davis, Jr sugirió la existencia de la heliosfera, la región del espacio que rodea el Sol formado por la interacción del medio interestelar local con el viento solar [34, 35]. Después de la detección del viento solar por la espacionave Mariner 2, otras misiones espaciales como las espacionaves Pionero (Pioneer) 10-11 y Viajero (Voyager) 1-2 exploraron la heliopausa y el medio interestelar. En 1993 Gurnett reportó por primera vez evidencia de la heliopausa basada en radio emisiones de $2-3 \mathrm{kHz}$ que venían de la heliopausa y detectadas por el Voyager 1-2 [34].

\section{Origen del Viento Solar}

El Sol presenta varios fenómenos magnéticos y estos son generados por el dinamo solar. Este es responsable por la actividad magnética en diferentes escalas temporales y espaciales [36, 37]. El Sol es considerado un cuerpo estratificado compuesto por un núcleo, una zona radiativa, zona convectiva, fotosfera, cromosfera, región de transición y corona. La radiación electromagnética es originada en el núcleo y viaja a través de la zona radiativa, que es la región que circunda el núcleo, hasta alcanzar la superficie solar. La zona convectiva es la capa donde la energía es transportada principalmente por convección [1, 2, 38, y referencias allí.

La fotosfera es llamada la superficie del Sol, allí se pueden observar manchas solares, fáculas y también la parte superior de las células de la zona convectiva. Encima de la fotosfera se encuentra la cromosfera, en esta región se pueden observar filamentos, prominencias y playas (plagues) cerca de las manchas solares. La región de transición es la capa que separa la cromosfera y la corona solar; allí la temperatura se incrementa 


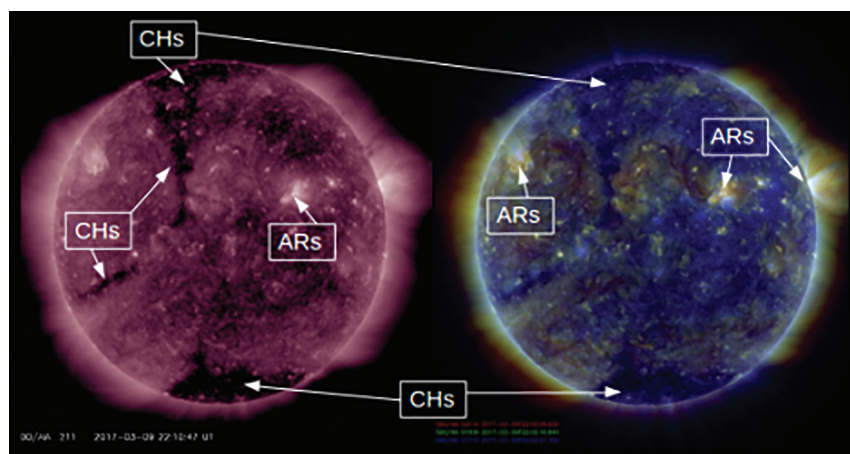

Figura 4: Huecos coronales Coronal Holes ( $\mathrm{CHs}$ ) y regiones activas solares (ARs) sobre la atmósfera solar, adaptada desde AIA/SDO images 2017-03-09 (YY-MM-DD), 22: 10:47 UT. Panel izquierdo: imagen del instrumento $A I A$ abordo del satélite SDO en 211A. Panel derecho: imagen compuesta por las longitudes de onda 211A, 193A y 171A. Fuente: Autora.

abruptamente hasta alcanzar millones de grados Kelvin en la corona que es la capa más externa del Sol. La corona puede ser observada durante los eclipses o usando cronógrafos, estos instrumentos cubren la superficie del Sol, permitiendo observar la corona. La corona solar, presenta diferentes configuraciones de campo magnético, con regiones de campo magnético abiertas y cerradas. Las regiones cerradas confinan plasma mientras las regiones abiertas transportan plasma a la heliosfera y al medio interplanetario. A lo largo de las líneas de campo abierto el plasma es transportado, permitiendo que partículas cargadas escapen de la atmósfera solar [1. 2, 38, y referencias allí.

Los huecos coronales (Coronal Holes ( $\mathrm{CHs}$ ) su nombre en inglés) están asociados con líneas de campo abierto, estas son las regiones donde el viento solar se origina (Figura 4). Este posee dos regímenes: el viento solar rápido asociado a huecos coronales $(\mathrm{CHs})$ en las regiones polares del Sol, con velocidades de $700-800 \mathrm{~km} / \mathrm{s}$ y el viento lento asociado a huecos coronales $(\mathrm{CHs})$ ubicados en las regiones ecuatoriales, poseen velocidades de $400 \mathrm{~km} / \mathrm{s}$ [39]41.

El viento solar posee flujos de iones pesados en varios estados, incluyendo Hidrógeno $\left(\mathrm{H}^{+}\right)$, Helio $\left(\mathrm{He}^{+}\right)$y Helio $\left(\mathrm{He}^{++}\right)$o partículas alfa. Los iones de helio se encuentran en magnetosferas planetarias y son usados como trazadores de la presencia de viento solar [42.

El viento solar continuamente se expande en el espacio interplanetario, su interacción con el campo magnético planetario o la ionosfera crea las magnetosferas planetarias [43, 44. La magnetosfera actúa como un escudo que protege la Tierra de eventos externos. El tamaño de la magnetosfera es determinada por el balance de presiones entre el la presión dinámica del viento solar y la presión combinada del campo magnético del planeta y el plasma.

La presión dinámica del viento solar se puede describir como

$$
P_{s w}=m_{p} N v_{s w}^{2}
$$

donde $P_{s w}$ es la presión dinámica, $m_{p}$ es la masa del protón con un valor de $1.6726219 \times 10^{-27} \mathrm{~kg}$, N es la densidad numérica del viento solar, $v_{s w}$ es la velocidad del viento solar.

La presión magnética se define como

$$
P_{B}=\frac{B^{2}}{8 \pi}
$$

donde $P_{B}$ es la presión magnética y $\mathrm{B}$ es el campo magnético.

Como la presión dinámica varía con el tiempo también lo hace el tamaño de la magnetosfera. El flujo del viento solar alrededor de la magnetosfera y el campo magnético planetario forma la cola magnética [40, 45.

El viento solar es fuertemente afectado por cambios en la actividad solar, este transmite el efecto de la variabilidad solar a los planetas. Debido a la acción de la rotación solar con un periodo de \pm 27 días, los patrones de campo magnético interplanetario muestran un comportamiento recurrente de 27 días. Además de las frecuentes variaciones de las propiedades del viento solar, esporádicos disturbios interplanetarios son causados por actividad solar como ondas de choque, eyecciones de masa coronal (CMEs) y llamaradas solares (flares) 34].

\section{Magnetosferas}

William Gilbert, físico de la Reina Elizabeth I, se dio cuenta 400 años atrás, que la Tierra se asemejaba a un imán [46]. Una visión más moderna describe que el campo magnético terrestre es generado por corrientes eléctricas fluyendo desde el interior de la Tierra. Descrito como un campo magnético dipolar se extiende más allá de la superficie del planeta, a través de la troposfera y dentro de la atmósfera ionizada, donde su efecto es considerable. La Figura 5 muestra esquemáticamente las capas de la atmósfera terrestre, una región ionizada de la atmósfera se encuentra entre la mesosfera y la exosfera, es llamada ionosfera [47,49]. Esta región es permanente ionizada debido a la fotoionización que provoca la radiación solar. Los procesos de ionización dependen de la actividad del Sol (por ejemplo, el período del ciclo solar/ciclo de manchas solares), el tiempo (cambios estacionales o diarios) y factores geográficos (zonas polares, de latitud media y ecuatoriales) [15].

El campo magnético terrestre afecta el movimiento de partículas ionizadas y así modifica las corrientes eléctricas ionosféricas y el movimiento de plasma. La importancia del campo magnético incrementa con la altitud, ya que la atmósfera llega a ser más escasa y su grado de ionización aumenta [50]. Estas densidades atmosféricas afectan directamente a los satélites de baja órbita debido al aumento de las fuerzas de arrastre en los satélites que propician su deterioro o decaimiento orbital [9, 51].

El término magnetosfera fue acuñado por T. Gold en 1959 para describir la región encima de la ionosfera en 


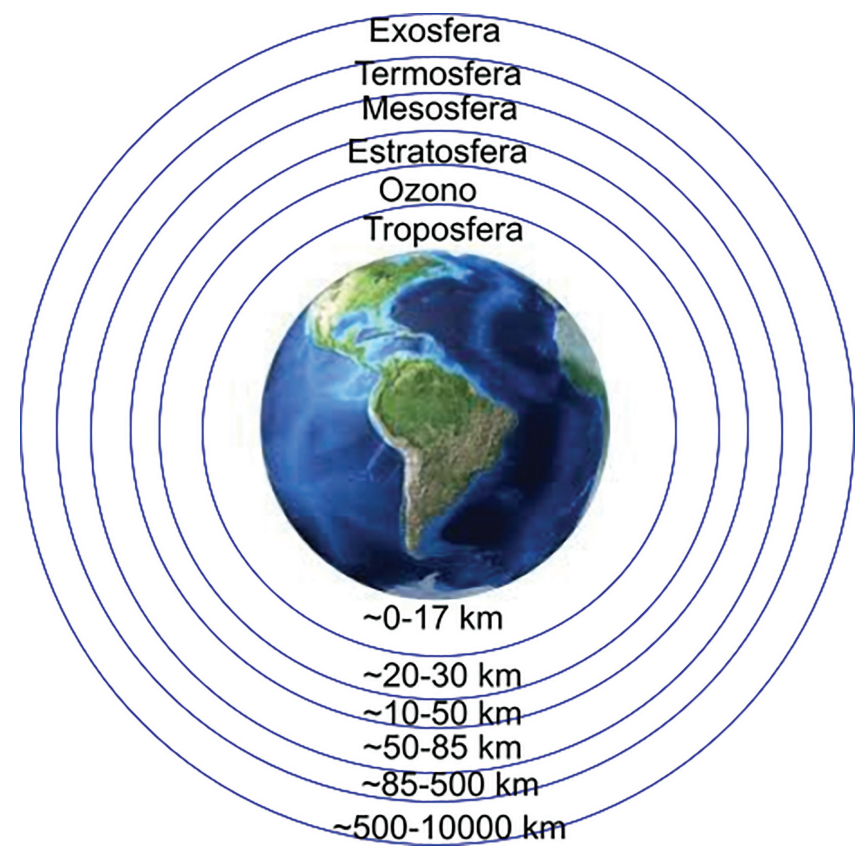

Figura 5: Diseño esquemático de las capas de la atmósfera terrestre. Fuente: Autora.

la cual el campo magnético de la Tierra controla los movimientos de partículas cargadas [26]. En el caso de la Tierra, cuando el viento solar interactúa con el campo magnético dipolar de la Tierra, su velocidad disminuye y se desvía alrededor del planeta, formando una cavidad llamada la magnetosfera. Los cinturones de Van Allen se encuentran en la magnetosfera terrestre donde se concentran partículas cargadas de alta energía (decenas de $\mathrm{KeV}$ y mayores), generadas por el viento solar y capturadas por el campo magnético terrestre. El control de estas partículas cargadas se extiende hasta $10 R_{T}$ ( $R_{T}$ radios terrestres) en dirección al Sol [44, [52, 53].

Cualitativamente, una magnetosfera planetaria es el volumen de espacio en el que el viento solar es excluido por el campo magnético del planeta. Sin embargo, en los planetas sin campo magnético, el viento solar que fluye crea cavidades cuyas propiedades son lo suficientemente similares a las de las magnetosferas reales. En un sentido más general la magnetosfera es una extensión de la atmósfera superior de un planeta en una región del espacio donde el campo magnético planetario domina (cuando el planeta posee campo magnético intrínseco y atmósfera) [44, 53.

En este caso la Tierra es usada como ejemplo para describir la magnetosfera globalmente. Sus principales regiones y características son descritas brevemente a continuación. El arco de choque (bow shock) es una importante región puesto que el viento solar interactúa con las líneas de campo terrestre en el lado diurno, la región detrás del arco de choque es conocida como una envoltura magnética (magnetosheath). La presión cinética del viento solar modifica la configuración exterior del campo magnético dipolar de la Tierra; en el

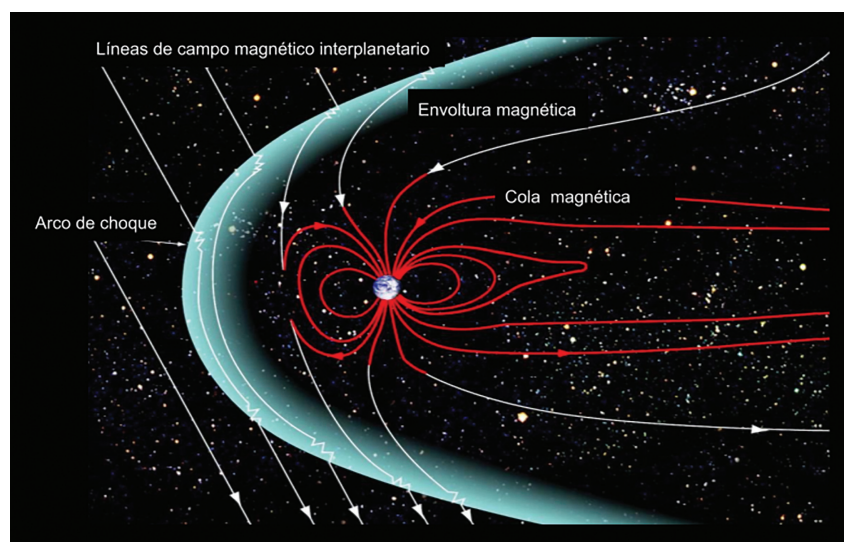

Figura 6: Magnetosfera terrestre, se puede observar el arco de choque (Bow shock), líneas de campo magnético interplanetario, la envoltura magnética (magnetosheath) y la cola magnética (magnetotail). Las líneas rojas muestran las líneas de campo magnético dipolar. Fuente: Adaptada de [54].

lado de dia de la magnetosfera el campo se comprime, mientras que en la magnetosfera del lado nocturno el campo se estira en una cola magnética (magnetotail) (Figura 6] 34.

Los cinturones de radiación de Van Allen forman una región en forma de toroide alrededor de los planetas conteniendo partículas energéticas. Esos cinturones tienen electrones e iones energéticos que se mueven a lo largo de las líneas de campo y oscilan entre los dos hemisferios [11, 53].

El sistema solar está dominado por el Sol, que forma su propia estructura magnética denominada heliosfera, tanto su tamaño como su estructura es gobernada por el movimiento relativo del Sol. La densidad del viento solar disminuye como el inverso del cuadrado de la distancia desde el Sol, así el plasma llega a ser suficientemente tenue. Entonces la presión del plasma interestelar impide su expansión. El viento solar disminuye su velocidad abruptamente a través de un Choque (conocido como el choque de terminación) antes de alcanzar la heliopausa, el límite que separa el viento solar del plasma interestelar [34, 44].

Las magnetosferas de planetas y lunas no magnetizadas pero con atmósfera como Venus, Marte y Titán, lunas no magnetizadas y sin atmósferas como la luna terrestre y magnetosferas de planetas magnetizados como Mercurio, Tierra, Júpiter, Saturno, Urano y Neptuno [19, 53].

\subsection{Magnetosferas de planetas magnetizados}

Las magnetosferas están directamente relacionadas con el campo magnético del planeta, su rotación y la interacción con el viento solar. Así las magnetosferas de cada planeta deben su tamaño a la magnitud del campo magnético que poseen, por ejemplo Júpiter y Saturno poseen un campo magnético aproximadamente 
dipolar con inclinación de $9.6^{\circ}$ y $0^{\circ}$ respectivamente. Urano y neptuno poseen campo magnético multipolar con inclinación de $59^{\circ}$ y $47^{\circ}[19$.

\subsubsection{Neptuno y Urano}

Ambos planetas poseen un campo magnético no simétrico, acoplado con la rápida rotación (Neptuno $\sim 16$ hrs y Urano $\sim 17$ hrs) y la inusual inclinación de los ejes de rotación, implica que las magnetosferas de estos planetas sufren drásticas variaciones geométricas [55. 56]. Medidas (in-situ) muestran que Urano posee una única configuración entre el planeta y el viento solar. Sin embargo se desconoce cómo esta configuración puede ser modulada, así como la interacción entre la exosfera e ionosfera. Futuras misiones a Urano pueden ayudar a entender el acoplamiento entre viento solar-magnetosferaionosfera y cual es el rol de cada uno de ellos en las condiciones de clima espacial del gigante helado [57, 58].

La aurora de Urano es una de las más significativas evidencias de cómo el clima espacial alrededor de un planeta cambia (Figura 7). Una de las características que intrigan es la interacción de sus lunas y anillos, que básicamente es desconocida y puede afectar drásticamente el clima espacial en el planeta [19.

Neptuno es relativamente una fuente débil de emisiones aurorales [60, 61. El plasma en la magnetosfera de Urano tiene relativa baja densidad y su origen está relacionado con el viento solar principalmente, en Neptuno la distribución de plasma está relacionada con Tritón que es su mayor fuente [62]. Tritón es la más grande luna entre las 14 que se conocen $(2700 \mathrm{~km}$ de diámetro, similar a Europa la luna de Júpiter), posee nitrógeno y metano en su atmósfera. Así el clima espacial en Neptuno puede estar relacionado con la interacción entre Tritón (fuente de plasma) y la magnetosfera de Neptuno 63.

\subsubsection{Saturno}

Saturno es el segundo planeta más grande en nuestro sistema solar, seguido de Júpiter. Saturno es un

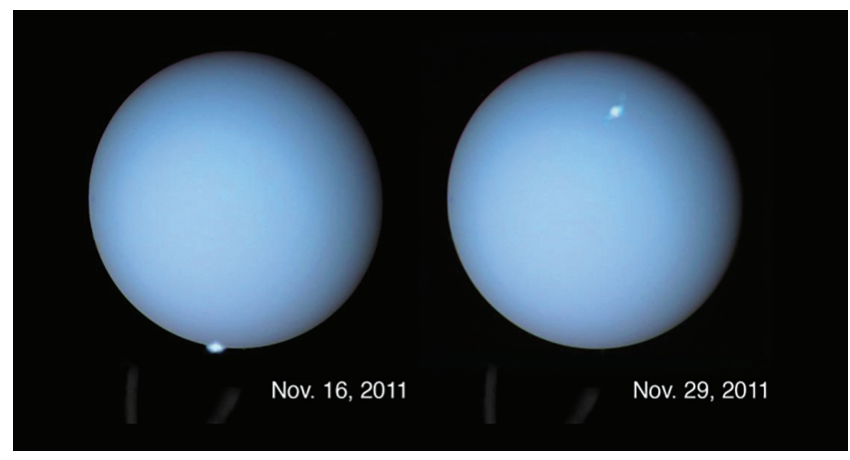

Figura 7: Auroras en el planeta Urano vistas por el telescopio espacial Hubble. Fuente: NASA, ESA, and L. Lamy (Observatory of Paris, CNRS, CNES) [59]. sistema con rápida rotación $\sim 10.5$ hrs [64]. El clima espacial en Saturno se manifiesta por la ocurrencia de aurora y emisiones termosféricas. La interacción del campo magnético de Saturno con el viento solar genera una gigante magnetosfera la cual esta dinámica y químicamente acoplada con las componentes del entorno de Saturno, esto es la interacción con los anillos, la exosfera, los satélites helados que poseen atmósferas tenues, las plumas de Enceladus y la atmósfera de Titán. Se cree que la magnetosfera de Saturno es un caso intermedio entre la magnetosfera de la Tierra y Júpiter 65, 66. Los otros satélites de Saturno se encuentran en la magnetosfera también y pueden actuar como fuentes o sumideros de plasma. Observaciones revelaron que el sistema de anillos es muy complejo, un ejemplo de esto es una ionosfera de los anillos detectada por la sonda espacial Cassini 67].

Las emisiones aurorales en Saturno pueden estar asociadas con la interacción del viento solar y la magnetosfera (Figura 8), similar a lo que ocurre con la aurora en la Tierra. Observaciones de la sonda Cassini y el telescopio espacial Hubble así lo han sugerido. El viento solar influencia la cola magnética en la magnetosfera de Saturno y esta interacción genera una huella en la aurora 69 71. La modulación de la aurora por viento solar fue reconocida a través de observaciones asociadas a emisiones en radio, observaciones del Voyager mostraron que la intensidad de la radiación kilométrica proveniente de Saturno (saturn kilometric radiation $S K R$ ) está correlacionada con la presión dinámica del viento solar 72 .

Uno de los satélites más importantes es Titán, este posee una atmósfera densa compuesta casi en su totalidad por Nitrógeno molecular. La misión Cassini demostró que la atmósfera de Titán es una fábrica

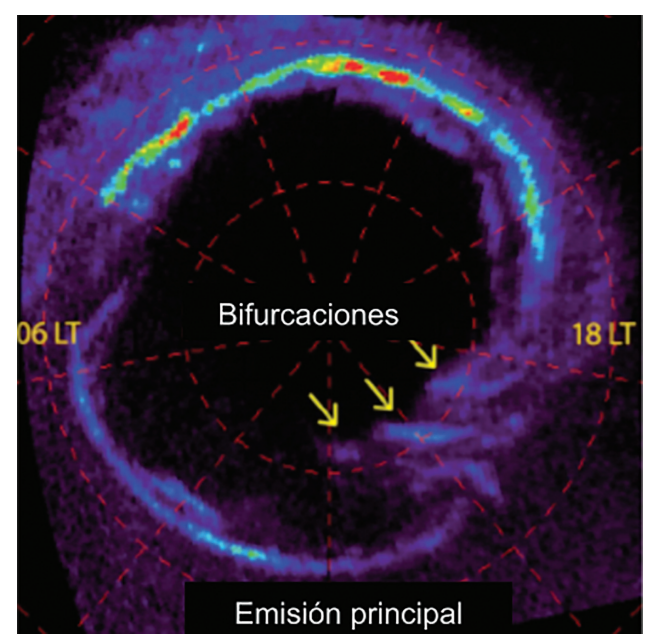

Figura 8: Aurora en Saturno observada con el instrumento UVIS a bordo de la sonda Cassini en DOY 21, 2009. Se observa la emisión principal y las bifurcaciones debidas a las interacciones con sus satélites. 06 LT y 18 LT se refieren al tiempo local (LT). Adaptada de [68]. 
química de formación de complejos iones positivos y negativos contribuyendo a una fuerte interacción entre la magnetosfera, ionosfera y atmósfera 73. Sin embargo la interacción entre Titán y el viento solar es similar a lo que ocurre en planetas sin campo magnético intrínseco como Marte y Venus 74. La helada luna Enceladus genera un sistema acoplado de corrientes que conecta la magnetosfera polar de norte a sur, dejando su huella en las emisiones aurorales también.

\subsubsection{Júpiter}

Júpiter posee un campo magnético 10 veces más grande que el de la Tierra, como resultado su magnetosfera se extiende hasta $150 R_{J}\left(R_{J}=71492 \mathrm{~km}\right)$ y sus cinturones de radiación son los más fuertes en el sistema solar. La variabilidad del plasma en el sistema joviano está determinada fuertemente con la actividad volcánica de la luna Io. Esta actividad tiene un efecto directo en la ionosfera, los cinturones de radiación y las exosferas de sus lunas. Aunque el viento solar también juega un papel en estas interacciones [19, 75].

Las fuentes internas de plasma de Júpiter como el vulcanismo de la luna Io y la rápida rotación son los principales factores en la dinámica de la aurora en Júpiter. Sin embargo, el viento solar juega un papel importante también en este proceso (Figura 99. Su magnetosfera gigante es una trampa y un acelerador de partículas energéticas 53 .

\subsection{Magnetosferas de planetas no magnetizados}

Mercurio poseen momento magnético relativamente pequeño $\sim 0.63 \times 10^{-3} M_{T}$. Las observaciones sugieren que la magnetosfera de Mercurio es muy pequeña para mantener plasma aprisionado alrededor del planeta. Además posee una rotación muy lenta, la fracción de momento dipolar más la alta presión dinámica del viento solar resultan en una magnetosfera muy pequeña. Mercurio no tiene una ionosfera significativa, sin embargo observaciones de la sonda Mariner-10 en 1973 y 1975; y la

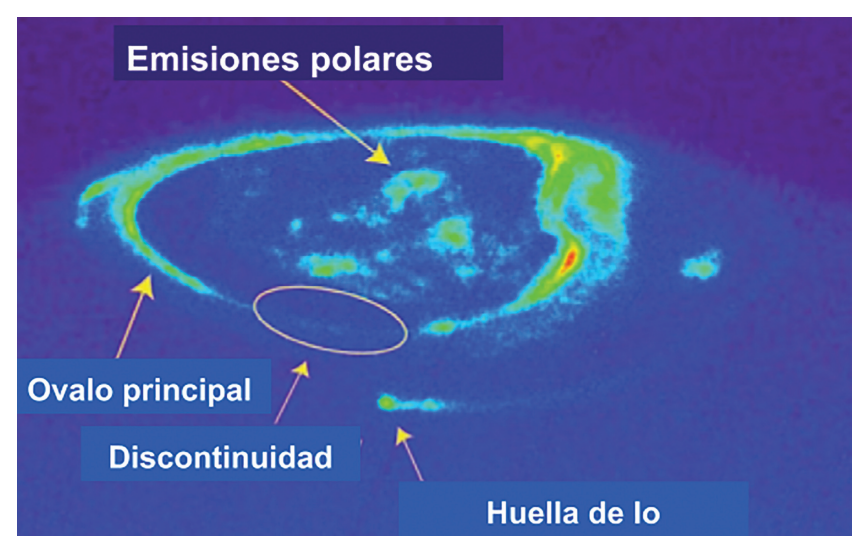

Figura 9: Aurora en Júpiter vista por el telescopio espacial Hubble en Diciembre 28 (2000). Fuente: Adaptada de [76]. sonda Messenger en 2008 muestran que la magnetosfera es dinámica, presentando variaciones en el flujo de partículas energéticas siendo posible ocurrencia de subtempestades [19, 53, 77, 78.

Venus presenta rotación lenta y en sentido opuesto del resto de los planetas. No posee un campo magnético intrínseco. El viento solar interactúa directamente con la ionosfera de Venus produciendo un frente de choque y una cola magnética bien definidas [53, 74, 79].

Marte posee una interacción con el viento solar similar a la de Venus y no posee campo magnético intrínseco, pero algunas partes de la crosta planetaria son magnetizadas y reversiones de polaridad del campo magnético pueden haber ocurrido cuando el dinamo planetario aún era activo. El viento solar puede interactuar con la atmósfera y las regiones de magnetización local. Así como Venus, Marte posee un frente de choque generado por la interacción del viento solar [53, 80].

\section{Clima Espacial Extremo en la Tierra}

Los eventos solares extremos pueden originarse en la corona solar (capa externa del Sol), en el viento solar, la magnetosfera, ionosfera y atmósfera terrestre. En la corona solar podemos observar las eyecciones de masa coronal $C M E s$. Algunos factores son importantes de resaltar, estas $C M E s$ extremas son masivas $\left(5 \times 10^{13} \mathrm{~kg}\right)$, poseen velocidades que llegan a alcanzar $\geq 1000 \mathrm{~km} / \mathrm{s}$ [81, llevan consigo intensos campos magnéticos $(100 n T)$ [82]. Los eventos extremos en el viento solar son extensiones de lo que ocurre en la corona solar. Sin embargo, estos incrementan debido a condiciones preexistentes, cuando otros CMEs han ocurrido en cortos intervalos de tiempo pre acondicionando la heliosfera o interactuando entre ellos, reteniendo momento e inyectando energía mayor en la magnetosfera [81. Eventos extremos en la magnetosfera son con frecuencia conocidos como tormentas geomagnéticas extremas y sus consecuencias pueden ser severas, con el gran número de satélites orbitando las fronteras de la magnetosfera [82.

Las variaciones ionosféricas pueden causar inducción de corrientes eléctricas en la Tierra, afectar los sistemas de distribución de energía, largos cables de comunicación, oleoductos, la reflexión, propagación y atenuación de señales inalámbricas, radio y televisión; interferencia en la señal de satélite, transmisiones, telecomunicaciones comerciales y sistemas GPS. Por otro lado las variaciones del campo magnético pueden afectar el control de altitud de naves espaciales y la navegación (brújulas). Las rafagas solares en radio interfieren con los radares y generan exceso de ruido en los sistemas de comunicaciones inalámbricos. En cuanto a la radiación de partículas esta causa daños en paneles solares y semiconductores, puede también afectar a los astronautas y pasajeros de aeronaves. En la atmósfera puede ocasionar atenuación y dispersión de señales inalámbricas 34,83 . 


\subsection{Ejemplos de clima espacial extremo}

Los fenómenos extremos ocurren durante episodios de aumento en la dinámica de la magnetosfera: tormentas geomagnéticas, auroras y precipitación de partículas que tienen gran importancia en el clima espacial.

A mediados del siglo XIX los telégrafos eléctricos revolucionaron las comunicaciones. Sin embargo, los telégrafos podían sufrir interrupciones en su funcionamiento debido a descargas eléctricas de los rayos. Procesos de clima espacial originados en el Sol producen corrientes telúricas o corrientes de tierra, este tipo de corrientes fueron nemesis para las primeras líneas de telégrafo. Un caso reportado por Barlow (1849) describe una aurora visible el 19 de Mayo de 1847 que afectó las agujas del telégrafo. Esta observación y su posible conexión entre un fenómeno natural y la nueva tecnología fue confirmado dramáticamente en Septiembre de 1859 durante el máximo del ciclo solar 10. Observado por Richard Carrington (Figura 10), una fulguración solar que fue el primer evento documentado como un evento extremo de clima espacial [28, 84, 85, ocasionando una intensa aurora que afectó varias estaciones de telégrafo desde el este de Estados Unidos, Inglaterra, Escandinavia, Bélgica, Francia, Suiza, Prusia, Austria y la Toscana [82, 83].

Con la invención de las comunicaciones inalámbricas, llevó al descubrimiento de la ionosfera. Sin embargo, las comunicaciones transoceánicas y oceánicas estuvieron llenas de frustraciones durante las dos primeras décadas del siglo 20. La razón principal fue el uso de una baja frecuencia haciendo posible que las comunicaciones se llevarán a cabo solamente durante la noche, cuando la radiación solar UV y los rayos X no ionizan la atmósfera alta, pero en ese momento esos efectos inducidos por el Sol no fueron identificados ni entendidos [34, 83].

Los primeros efectos significativos de los procesos entre el Sol y la Tierra sobre las tecnologías ocurrió durante el ciclo solar 17, en Marzo de 1940 una gran tormenta

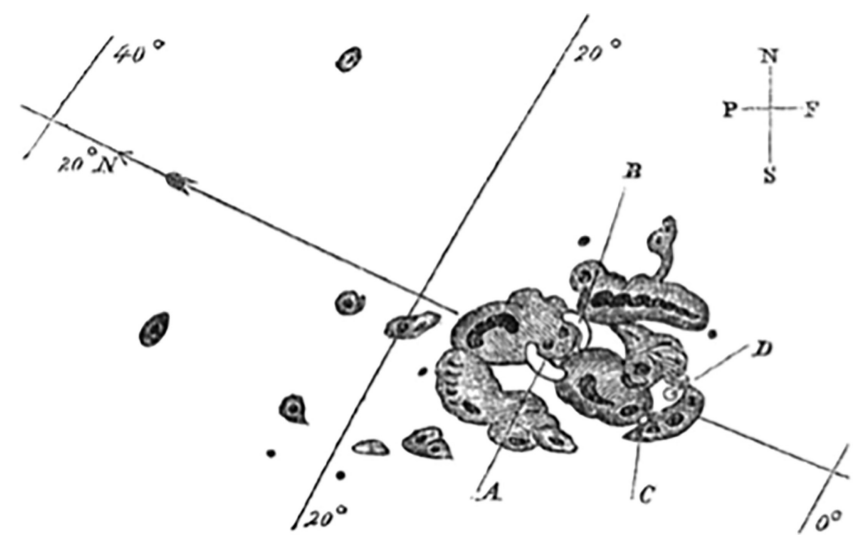

Figura 10: Evento Carrington dibujado a mano (Carrington 1859), los puntos A y B marcan las posiciones iniciales de una intensa fulguración que después de aproximadamente cinco minutos se movió a los puntos $C$ y $D$, para después desaparecer. Fuente: [84.

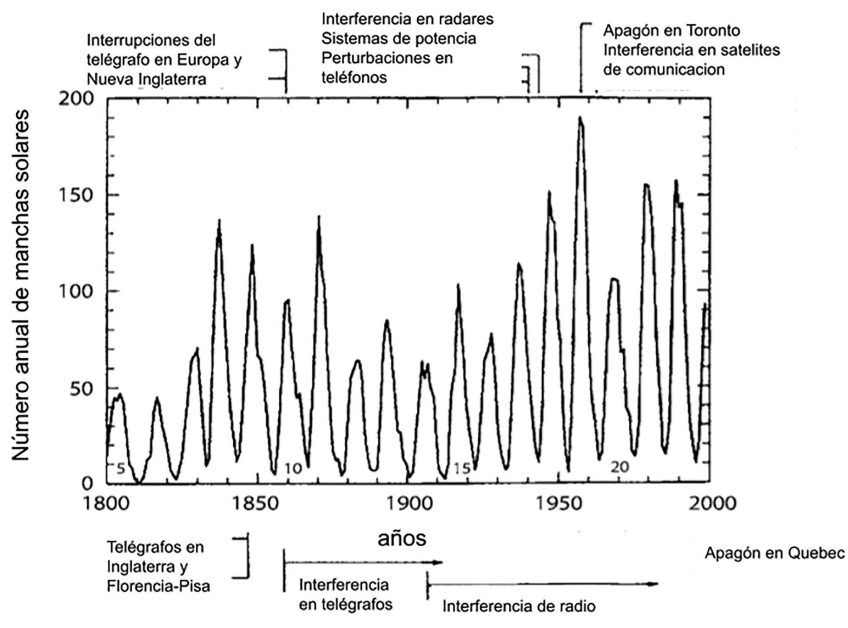

Figura 11: Números anuales de manchas solares y algunos de los más importantes impactos de la actividad solar en la Tierra. Fuente: Adaptado de [34].

geomagnética causó problemas en los sistemas de energía eléctrica en la región de Ontario y el norte de los Estados Unidos (Figura 11). Durante el ciclo solar 17 también se descubrió como intensas emisiones solares en radio interfirieron con un radar que se estaba utilizando en el Reino Unido para proporcionar advertencias de aviones alemanes durante la Segunda Guerra Mundial [86. Fue durante el mismo evento que se hicieron las primeras mediciones de partículas solares desde la Tierra [87.

El día de la bastilla Julio 14, 2000 cerca al máximo del ciclo solar 23 ocurrió una llamarada solar de clasificación X5.7, seguido de una eyección de masa coronal (CME), que generó una gran tormenta geomagnética (Figura 12 89]. Octubre de 2003 fue un periodo de extraordinaria actividad solar, grandes llamaradas en rayos X de clasificación X17 y X10 acompañadas de eyecciones de masa coronal (CMEs) entre el 28 y 29 de Octubre (Figura 13) que ocasionaron una gran tormenta geomagnética, durante este periodo las comunicaciones de alta frecuencia fueron imposibles, entre otros efectos en la ionosfera. Estos eventos ayudaron a justificar una

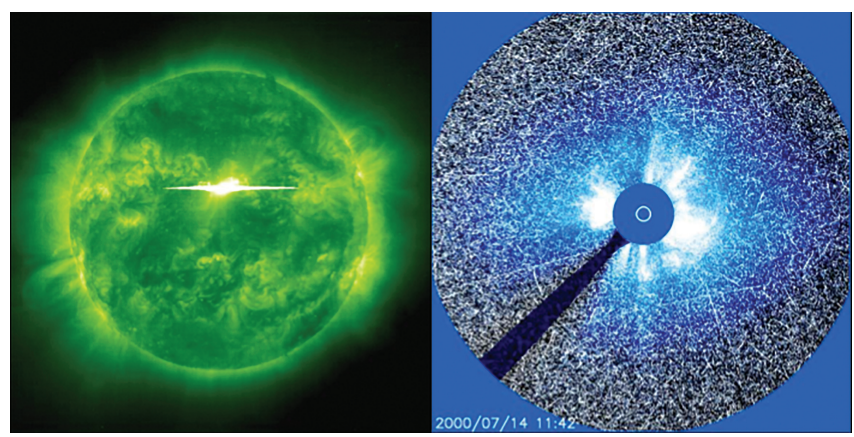

Figura 12: Evento del día de la bastilla Julio 14, 2000, visto por el satélite SOHO (panel izquierdo) ocasionó una tormenta de partículas y una CME dirigida hacia la Tierra visto en el coronógrafo LASCO C3 (panel derecho). Fuente: [88]. 


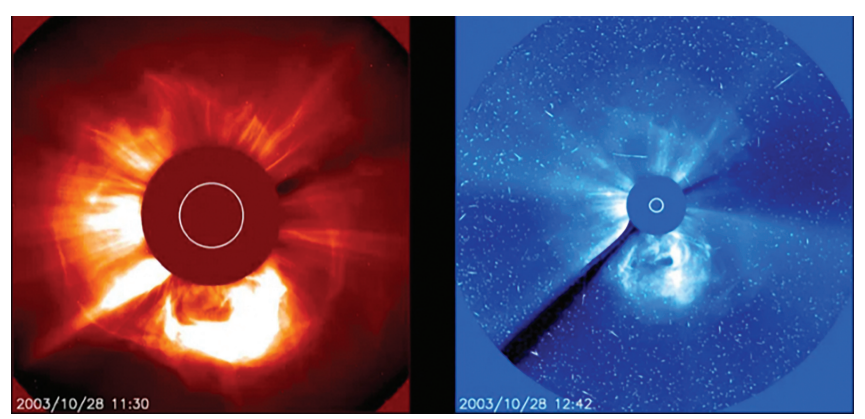

Figura 13: Evento del día de Halloween Octubre 28 2003, eyección de masa coronal visto en el coronógrafo LASCO C2 (panel izquierdo) y C3 (panel derecho). Fuente: [90].

significante inversión en observaciones que ayuden a entender el clima espacial [91].

La influencia de estos factores en la tecnología espacial es conocida, un ejemplo de esto fue el detector de radiación del Mars Radiation Environment Experiment (MARIE) abordo del la nave Mars Odyssey, la cual se presume fallo debido a un evento de particulas energeticas solares (SEP) ocurrido entre Octubre y Noviembre de 2003 [92].

\section{2. ¿Cómo monitorear el clima espacial?}

En la actualidad podemos monitorear la actividad geomagnética a escala global a través de la variación del campo magnético de la Tierra o actividad geomagnética 93. Para esto se han desarrollado índices como Dst, Kp, AL, AU y AE que permiten monitorear la actividad geomagnética relacionada con la actividad solar.

El índice Dst ("Disturbance storm time"), es una medida estándar de la intensidad de una tormenta geomagnética: mientras más negativo es el valor del índice Dst, la tormenta es más intensa 94. El índice Dst es un promedio por hora del campo magnético medido en el suelo, es calculado mensualmente usando datos desde diferentes estaciones equipadas con magnetómetros alrededor del globo (Figura 14). El índice Dst es altamente correlacionado con la componente hacia el sur de campo magnético $B_{z}(t)[34,95$, debido a la ocurrencia de la reconexión magnética 96 ].

El índice $\mathrm{K}$ planetario $(\mathrm{Kp})$, se utiliza para caracterizar la magnitud de las tormentas geomagnéticas, valores $>4$ caracterizan tormentas geomagnéticas más intensas. Este índice describe las perturbaciones en el campo magnético de la Tierra y es usado para decidir si es necesario emitir alertas y advertencias geomagnéticas para los usuarios afectados por estas perturbaciones 5 Los principales efectos se dan sobre la red eléctrica, los usuarios de señales de radio que se reflejan en la ionosfera o que pasan a través de ella y los observadores de la aurora 97 .

\footnotetext{
5 https://www.swpc.noaa.gov/products/planetary-k-index
}
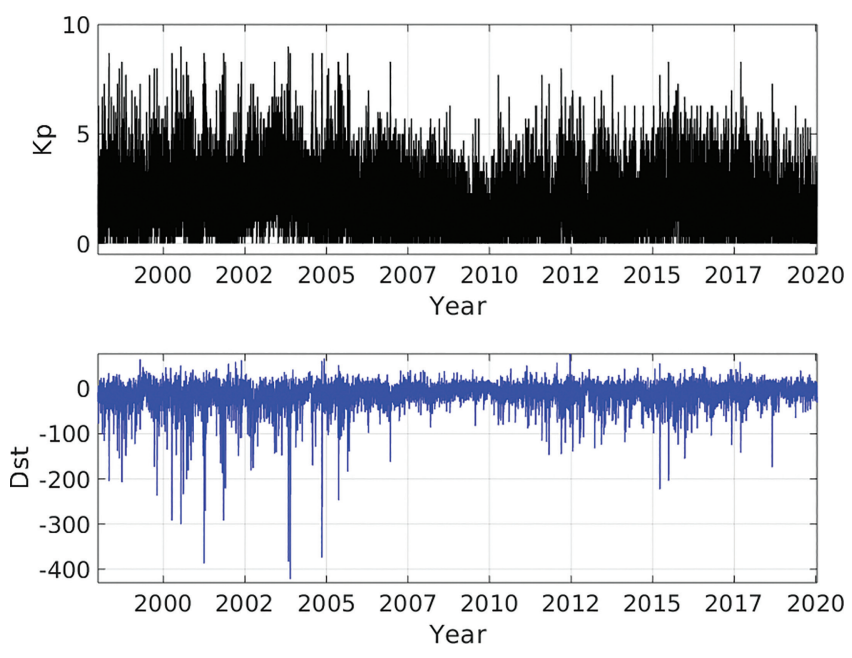

Figura 14: Series de tiempo de los índices geomagneticos desde 1998-01-01 hasta 2020-30-23 (base de datos de OMN 6 ), índice Kp (panel superior) e índice Dst (panel inferior).

Los índices AE, AU y AL fueron diseñados por T. N. Davis y M. Sugiura en 1966. Estos índices describen el nivel de perturbación registrado por los magnetómetros de la zona auroral. Las envolventes superior e inferior de esta superposición definen los índices AU (amplitud superior) y AL (amplitud inferior), respectivamente. La diferencia entre las dos envolventes determina el índice $\mathrm{AE}$ (Electrojet Auroral), es decir, $\mathrm{AE}=\mathrm{AU}-\mathrm{AI}\left[{ }^{7}[11\right.$.

El estudio del clima espacial en otros planetas es un proceso aún más complejo, datos de las espacionaves pueden generar información cuando estas se aproximan a los planetas, pero no contamos con un monitoramento constante como ocurre en la Tierra. Sin embargo, las simulaciones MHD pueden ser una gran herramienta para entender procesos de clima espacial en otros planetas. Existe un gran número de simulaciones MHD para modelar la magnetosfera de Júpiter [98101 y la magnetosfera de Saturno 102 104. El modelo mSWiM es un ejemplo, este modelo usa datos de viento solar propagado hasta los planetas usando una descripción magnetohidrodinámica $(\mathrm{MHD}){ }^{8}$ [105, 106]. Los datos han sido validados usando datos de Pioneer, Voyager, Ulysses y Cassini. Este modelo describe el viento solar a través de parámetros como la velocidad $(v(\mathrm{~km} / \mathrm{s}))$, campo magnético $(B(n T))$, densidad numérica $\left(N\left(\# \mathrm{~cm}^{-3}\right)\right)$ donde \# corresponde al número de partículas y temperatura $(\mathrm{T}(\mathrm{K}))$ (Figura 15).

El uso de medidas in situ obtenidas por las espacionaves como Voyager, Galileo, Cassini, Ulyses, New Horizons y Juno, permiten observar de cerca variaciones en la magnetosfera de algunos planetas, un ejemplo son las auroras polares. Algunas de estas espacionaves permiten medir también variaciones en el campo magnético

\footnotetext{
6 https://omniweb.gsfc.nasa.gov/html/ow_data.html

7 https://ccmc.gsfc.nasa.gov/modelweb/solar/ae.html

8 http://mswim.engin.umich.edu/
} 

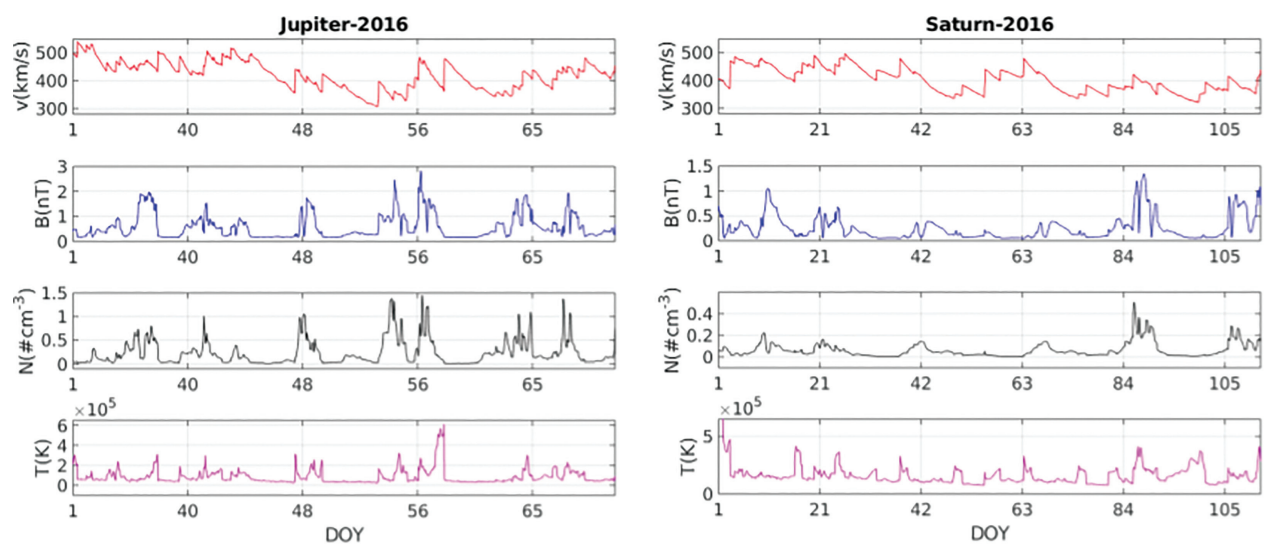

Figura 15: Variaciones de viento solar en Júpiter (panel izquierdo) y Saturno (panel derecho) en 2016 usando datos del modelo mSWiM.

y evaluar las variaciones debidas al viento solar. Pero la combinación de estas medidas y los modelos pueden permitir una mejor comprensión de los fenómenos de clima espacial en planetas, generando una razonable descripción de la estructura en gran escala y la dinámica de las magnetosferas planetarias.

\section{Discusión}

La naturaleza de la actividad solar y sus efectos en la magnetosferas e ionosféras son muy importantes en nuestra sociedad que cada día depende más de la tecnología. Pero ¿cuáles son las consecuencias de los eventos extremos de clima espacial? desde la última década se ha reconocido la importancia e interdependencia de varios componentes de nuestra compleja y tecnológica sociedad. Interrupciones de energía eléctrica y suministros de petróleo/gas pueden tener un efecto de cascada significante en un amplio rango de sectores, incluyendo bancos, finanzas, servicios de gobierno y respuestas de emergencia 82. Una de las más controversiales y potenciales consecuencias de una tormenta geomagnética extrema puede conllevar a pérdidas de 1-2 trillones de dólares, 130 millones de personas sin energía eléctrica por varios años, debido a la destrucción de cientos de transformadores [107]. Tormentas como la de marzo de 1989, dan una idea de cómo la tecnología y la red eléctrica, en particular, podrían verse afectadas. Una tormenta geomagnética más reciente en Octubre de 2003 (llamada "tormenta de Halloween"), por ejemplo, pueden haber causado la destrucción de cinco transformadores y el daño de diez más en Sudáfrica [108. Sin embargo, inferir las probables consecuencias de un evento extremo de clima espacial es un camino incierto aún. Nuestra experiencia se limita a un puñado de eventos, necesitamos un estudio en largas escalas de tiempo que permita describir mejor estos posibles escenarios y prepararnos para ellos.

\section{REFERÊNCIAS}

[1] J.M. Rodríguez, El Sol y la irradiancia. El Sol: conceptos básicos (Editorial Académica Española, Chisinau, 2018).

[2] J.M. Rodríguez Gómez, F. Carlesso, L.E. Vieira y L. Silva, Revista Brasileira de Ensino de Física 40, e3312 (2018).

[3] E. Echer, N. Rigozo, D. Nordemann, L.E. Antunes Vieira, A. Prestes y H. De Faria, Revista Brasileira de Ensino de Física 25, 157 (2003).

[4] J.A. Eddy, Science 192, 1189 (1976).

[5] J.T. Gosling, en: Coronal Mass Ejections, Geophysical Monograph 99, editado por N. Crooker, J.A. Jocelyn y J. Feynman (American Geophysical Union, Washington, 1997).

[6] N. Gopalswamy, S. Yashiro, G. Michalek, G. Stenborg, A. Vourlidas, S. Freeland y R. Howard, Earth, Moon, and Planets 104, 295 (2009).

[7] O.R. Nelson, Revista Brasileira de Ensino de Física 35, 3317 (2013).

[8] A. Balogh, V. Bothmer, N.U. Crooker, R.J. Forsyth, G. Gloeckler, A. Hewish, M. Hilchenbach, R. Kallenbach, B. Klecker, J. Linker et al., Space Science Reviews 89, 141 (1999).

[9] D.M. Oliveira y M.V.D. Silveira, Revista Brasileira de Ensino de Física 39, e3305 (2017).

[10] E. Costa Jr., F.J.R. Simões Jr., F.R. Cardoso y M.V. Alves, Revista Brasileira de Ensino de Física 33, 4301 (2011).

[11] E. Costa Jr., F.R. Cardoso, F.J.R. Simões Jr. y M.V. Alves, Revista Brasileira de Ensino de Física 33, 2302 (2011).

[12] M. Moldwin, An Introduction to Space Weather (Cambridge University Press, Cambridge, 2008).

[13] R. Schwenn, Living Review in Solar Physics 3, 2 (2006).

[14] E. Echer, M. Alves y W. Gonzalez, Revista Brasileira de Ensino de Física 28, 51 (2006).

[15] O. Novik, F. Smirnov y M. Volgin, Electromagnetic Geophysical Fields (Springer, Cham, 2019), c. 4, p. 61. 
[16] P.P. Ferreira, M.V.D. Silveira, F.R. Cardoso, D. Koga V.M.C.S. Souza, L.E.A. Vieira, G. Fariñas Pérez, B.M. Cuadros-Melgar, W.D. Gonzalez y E.F. Santos, Revista Brasileira de Ensino de Física 39, e2307 (2017).

[17] R.P. Kane, Advances in Space Research 37, 1261 (2007).

[18] J. Lilensten y A. Belehaki, Acta Geophysics 57, 1 (2009).

[19] C. Plainaki, J. Lilensten, A. Radioti, M. Andriopoulou, A. Milillo, T. Nordheim, J. Dandouras, A. Coustenis, D. Grassi, V. Mangano et al., J. Space Weather Space Climate 6, A31 (2016).

[20] L. Biermann, The observatory 77, 109 (1957).

[21] R.P. Kane, Advances in Space Research 44, 1252 (2007).

[22] M. Glenn Sterenborg, O. Cohen, J.J. Drake y T.I. Gombosi, Journal of Geophysical Research 116, A01217 (2011).

[23] E. Schatzman, en: Memoires de la Societe Royale Des Sciences de Liege, Cinguieme serie (Institut d'Astrophysique, Liège, 1959), t. 3, p. 295.

[24] P. Biermann, Astronomy and Astrophysics 22, 407 (1973).

[25] https://www.esa.int/ESA_Multimedia/Images/2019/ 03/Parker_spiral

[26] T. Gold, Journal of Geophysical Research 64, 1219 (1959).

[27] https://spaceplace.nasa.gov/aurora/en/

[28] D.M. Oliveira, Revista Brasileira de Ensino de Física 42, e20190213 (2020).

[29] K. Birkeland, The Norwegian aurora polaris expedition, 1902-1903 (H. Aschehoug, Oslo, 1908).

[30] I.G. Richardson, Living Reviews in Solar Physics 15, 1 (2018).

[31] P. Janardhan, S. Vadawale, B. Bapat, K.P. Subramanian, D. Chakrabarty, P. Kumar, A. Sarkar, N. Srivastava, T.R. Satheesh, V.K. Yada et al., Current Science 113, 620 (2017).

[32] J.T. Gosling, R.T. Hansen y S.J. Bame, Bulletin of the Astronomical Society 3, 267 (1971).

[33] M. Neugebauer y C.W. Snyder, Science 138, 1095 (1962).

[34] Y. Kamide y A. Chian (eds), Handbook of the solar terrestrial environment (Springer, Berlin, 2007).

[35] W.I. Axford, Space Science Reviews 78, 9 (1996).

[36] M.J. Aschwanden, Physics of the solar corona. An introduction with problems and solution (Springer, Berlin, 2005).

[37] H. Zirin, Astrophysics of the Sun (Cambridge University Press, New York, 1988).

[38] J.M. Rodríguez Gómez, Evolution of the electron density, temperature distribution in the solar corona during the solar cycles 23 and 24. Tesis de Doctorado, Instituto Nacional de Pesquisas Espaciais, São José dos Campos (2017).

[39] A.Z. Wafaa y A. Zainab, International Journal of Current Research and Academic Review 4, 77 (2016).

[40] R. Schwenn, Space Science Reviews 124, 51 (2006).

[41] M.G. Kivelson y C.T. Russell (eds), Introduction to Space Physics (Cambridge University Press, Cambridge, 1995).
[42] P.A. Delamere, F. Bagenal, C. Paranicas, A. Masters, A. Radioti, B. Bonfond, J. Ray, X. Jia, J. Nichols y C. Arridge, Space Science Review 187, 51 (2015).

[43] S.J. Bolton, F. Bagenal, M. Blanc, T. Cassidy, E. Chané, C. Jackman, X. Jia, A. Kotova, N. Krupp, A. Milillo et al., Space Sci Rev 192, 209 (2015).

[44] M.G. Kivelson y F. Bagenal, en: Encyclopedia of the Solar System, editado por L. McFadden, P.R. Weissman y T.V. Johnson (Academic Press, Cambridge, 2007), 2 ed., p. 519.

[45] J.D. Richardson, en: Heliophysical Processes. Astrophysics and Space Science Proceedings, editado por N. Gopalswamy, S. Hasan y A. Ambastha (Springer, Berlin, 2010), p. 83.

[46] W. Gilbert, On the Magnet and Magnetic Bodies, and on That Great Magnet the Earth (Dover, New York, 1991).

[47] D. Oliveira, Revista Brasileira de Ensino de Física 36 , 1305 (2014).

[48] L.S. Alperovich y E.N. Fedorov, Hydromagnetic Waves in the Magnetosphere and the Ionosphere (Springer, Dordrecht, 2007).

[49] H. Rishbeth y O.K. Garriott, Introduction to ionospheric physics (Academic Press, New York, 1969).

[50] J.K. Hargreaves, The solar-terrestrial environment. An introduction to geospace - the science of the terrestrial upper atmosphere, ionosphere and magnetosphere. Cambridge Atmospheric and Space Science Series (Cambridge University Press, Cambridge, 1995).

[51] E. Zesta y C.Y. Huang, en: Space Weather Fundamentals, editado por G.V. Khazanov (CRC Press, Boca Raton, 2016), p. 329.

[52] R.S. Dutra, D.S.R. Ferreira, A.S.M. Goncalves y G.M. Carvalho, Revista Brasileira de Ensino de Física 42, e20190164 (2020).

[53] E. Echer, Revista Brasileira de Ensino de Física 32, 2301 (2010).

[54] https://www.nasa.gov/mission_pages/sunearth/mult imedia/magnetosphere.html

[55] C. Guervilly, P. Cardin y N. Schaeffe, Icarus 218, 100 (2012).

[56] F. Herbert, J. Geophys. Res. 114, A11, 2009.

[57] D.Turrini, R. Politi, D. Peron, D. Grassi y C. Plainaki, Planet. Space Sci 104, 93 (2014).

[58] C.C. Arridge, N. Agnor, K.H. Andre y L.N. Baines, Exp. Agric. 33, 753 (2012).

[59] https://www.nasa.gov/mission_pages/hubble/science/ uranus-aurora.html

[60] P. Zarka, J. Geophys. Res 103, 20159 (1998).

[61] P. Zarka, P.B.M. Perdensen, A. Lecacheux, M.L. Kaiser, M.D. Desch, W.M. Farrell y W.S. Kurth, en: Neptune and Triton, editado por D.P. Cruikshank, M.S. Matthews y A.M. Schumann (Astronomisches RechenInstitute Publisher, Heidelberg, 1995), p. 341.

[62] J.D. Richardson, J.W. Belcher, M. Zhang y R.L. McNutt Jr., Journal of Geophysical Research 96, 18993 (1991).

[63] A. Masters, Journal of Geophysical Research 119, 5520 (2014).

[64] T.I. Gombosi, T.P. Armstrong, C.S. Arridge, K.K. Khurana, S.M. Krimigis, N. Krupp, A.M. Persoon 
y M.F. Thomsen, en: Saturn from Cassini-Huygens, editado por M.K. Dougherty, L.W. Esposito y S.M. Krimigis (Springer, Heidelberg, 2009).

[65] M. Blanc, S. Bolton, J. Bradley, M. Burton y T.E. Cravens, Space Sci. Rev 104, 253 (2002).

[66] M. Andriopoulou, N. Roussos, C. Krupp, M. Paranicas, S. Thomsen, S. Krimigis, M.K. Dougherty y K.H. Glassmeier, Icarus 229, 57 (2014).

[67] A.J. Coates, H.J. Mc Andrews, A.M. Rymer, D.T. Young y F.J. Crary, Gephys. Res. Lett 32, L14S09 (2005).

[68] A. Radioti, D. Grodent, J.C. Gerard, S.E. Milan, B. Bonfond, J. Gustin y W. Pryor, Journal of Geophysical Research 116, A11209 (2011).

[69] J.W. Dungey, Phys. Rev. Lett 6, 47 (1961).

[70] V.M. Vasyliunas, en: Physics of the Jovian magnetosphere, editado por A.J. Dessler (Cambridge University Press, Cambridge, 1983), p. 395.

[71] P.A. Delamere y F. Bagenal, Journal of Geophysical Research 118, 7045 (2013).

[72] M.D. Desch y M.L.Kaiser, Geophysics Research Letters 8, 253 (1981)

[73] J.H. Waite, R.V. Niemann, W.T. Yelle, T.E. Kasprzak y T.E. Cravens. Science 308, 982 (2005).

[74] C. Bertucci, F. Duru, N. Edberg, M. Fraenz, C. Martinecz, K. Szego y O. Vaisberg, Space Sci Rev 162, 113 (2011).

[75] E.J. Bunce, S.W.H. Cowley y T.K. Yeoman, Journal of Geophysical Research 109, A09S13 (2004).

[76] A. Radioti, J.C. Gérard, D. Grodent, B. Bonfond, N. Krupp y J. Woch, Journal of Geophysical Research 113, A01215 (2008).

[77] P. Diego, M. Piersanti, M. Laurenza y U. Villante, Journal of Geophysical Research: Space Physics 125, e28281 (2020).

[78] L.F. Burlaga, Planet Space Sci 49, 1619 (2001).

[79] H. Curry, J. Luhmann, J. Gruesbeck, Y. Ma, S. Bale y K. Goodrich, en EPSC Abstracts (Geneva, 2019).

[80] R. Ramstad, D.A. Brain, Y. Dong, J. Espley, J. Halekas y B. Jakosky, Nature Astronomy 4, 979 (2020).

[81] J.M. Rodríguez Gómez, T. Podladchikova, A. Veronig, A. Ruzmaikin, J. Feynman y A. Petrukovich, The Astrophysical Journal 899, 47 (2020).

[82] P. Riley, D. Baker, Y. Liu, P. Verronen, H. Singer y M. Güdel, Sci Rev 214, 21 (2018).

[83] L.J. Lanzerotti, Space Sci Rev 212, 1253 (2017).

[84] R.C. Carrington, Mon. Not. R. Astron. Soc. 20, 13 (1859).

[85] E.W. Cliver, Advances in Space Research 38, 119 (2006).

[86] J.S. Hey, Nature 157, 47 (1946).

[87] S.E. Forbush, Physical Review 70, 771 (1946).

[88] https://sohowww.nascom.nasa.gov/gallery/top10/

[89] S. Watari, M. Kunitake y T. Watanabe, Solar Physics 204, 425 (2001).

[90] https://www.thesuntoday.org/historical-sun/x17-sol ar-flare-and-solar-storm-of-october-28-2003/

[91] J.M. Goodman, Space weather and telecommunications (Springer, New York, 2005).

[92] V. Andersen, BAAS 38, 90 (2006).
[93] G. Rostoker, Reviews of Geophysics 10, 935 (1972)

[94] W.D. Gonzalez, J.A. Joselyn, Y. Kamide, H.W. Kroehl, G. Rostoker, B.T. Tsurutani y V.M. Vasyliunas, Journal of Geophysical Research 99, 5771 (1994).

[95] D.M. Oliveira y D.M. Silveira, Revista Brasileira de Ensino de Física 38, 1305 (2016).

[96] V.M. Souza, M.V.D. Silveira, D. Koga y P.R. Jauer, Revista Brasileira de Ensino de Física 38, e2301 (2016).

[97] D. Wagner y R. Neuhäuser, Astronomische Nachrichten 340, 483 (2019).

[98] K. Fukazawa, T. Ogino y R.J. Walker, Geophysics Research Letters 32, 103202 (2005).

[99] K. Fukazawa, T. Ogino y R.J. Walker, Journal of Geophysics Research 111, A10207 (2006)

[100] K. Fukazawa, T. Ogino y R.J. Walker, Geophysics Research 115, A09219 (2010).

[101] T. Moriguchi, A. Nakamizo, T. Tanaka, T. Obara y H. Shimazu, Journal of Geophysics Research 113, A05204 (2008).

[102] R.M. Wingle, A. Kidder, E. Harnett, N. Iflan, C. Paty y D. Snowden, Journal of Geophysics Research 118, 4253 (2013).

[103] X. Jia y M.G. Kivelson, Journal of Geophysics Research 117, A11219 (2012).

[104] R.J. Walker, K. Fukazawa, T. Ogino y D. Morozoff, Journal of Geophysics Research 116, A03203 (2011).

[105] B. Zieger y B.H. Kennth, Journal of Geophysical Research 113, A08107 (2008).

[106] G.A. Toth, Astrophysical Letters and Communications 34, 245 (1996).

[107] J.G. Kappenman, en: Electric Power Generation, Transmission, and Distribution, editado por L.L. Grigsby (CRC Press, Boca Raton, 2012), c. 16, 3 ed.

[108] D. McMorrow, Impacts of Severe Space Weather on the Electric Grid (JASON, Virginia, 2011). 